\title{
Regras para formação de grupos de colaboração utilizando detecção automática de traços de personalidade
}

\section{Rules for forming collaborative groups using automatic detection of personality traits}

\author{
Taís Borges Ferreira \\ Universidade Federal de Uberlândia \\ taisbferreira@ufu.br
}

\author{
Andrey Ricardo Pimentel \\ Universidade Federal do Paraná \\ andrey@inf.ufpr.br
}

\author{
José Antonio Buiar \\ Universidade Tecnológica \\ Federal do Paraná \\ buiar@utfpr.edu.br
}

\author{
Márcia Aparecida Fernandes \\ Universidade Federal de Uberlândia \\ marcia@ufu.br
}

\author{
Luiz Eduardo S. Oliveira \\ Universidade Federal do Paraná \\ luiz.oliveira@inf.ufpr.br
}

\begin{abstract}
Resumo
A formação de grupos é um aspecto crucial da aprendizagem colaborativa. Devido à falta de interação entre os alunos, essa tarefa torna-se complexa, e ferramentas que ajudam a determinar grupos para o trabalho colaborativo são necessárias. Propostas para detectar traços de personalidade e formar grupos, baseadas no modelo Big Five, foram desenvolvidas. Entretanto, esses trabalhos não apresentam regras para formação de grupos. Assim, este trabalho verifica a viabilidade de detectar automaticamente traços de personalidade através de textos escritos, $e$ demonstra a influência desses traços na formação do grupo, identificando um conjunto de regras para este fim. Além disso, este artigo é um esforço conjunto de dois grupos de pesquisa para identifica algoritmos adequados para deteç̧ão de traços de personalidade a partir de textos. As regras de agrupamento foram extraídas a partir de base de dados dos grupos construída, a fim de ajudar na formação de novos grupos. Portanto, as contribuições desta pesquisa foram ferramentas para deteç̧ão automática de traços de personalidade a partir de textos, identificação de algoritmos de aprendizagem mais adequados para classificação de traços, base de dados de grupos e um conjunto de regras baseadas em traços e outros parâmetros para formação de grupos.
\end{abstract}

Palavras-Chave: Modelo Big Five, traços de personalidade, formação de grupos, aprendizagem colaborativa

\begin{abstract}
Group formation is a crucial aspect of collaborative learning. Due to lack of interaction among students, this task becomes complex, and tools that determine groups for collaborative work are needed. Proposals for detecting personality traits and forming groups, based on the Big Five model, were developed. However, these works do not present rules for group formation. Thus, this work verifies the feasibility of automatically detecting personality traits through written texts and demonstrates the influence of these traits on group formation, identifying a set of rules for this purpose. In addition, this article is a joint effort of two research groups to identify suitable algorithms for detecting personality traits from texts. The grouping rules were extracted from the database of the groups built in order to help in the formation of new groups. Therefore, the contributions of this research were tools for automatic detection of personality traits from texts, identification of learning algorithms more suitable for classification of traits, database of groups and a set of rules based on traits and other parameters.
\end{abstract}

Keywords: Big Five Model, personality traits, group formation, collaborative learning

Cite as: Ferreira, T. B., Buiar, J.A., Fernandes, M. A., Pimentel, A. R. \& Oliveira, L. S. (2020). Rules for forming collaborative groups using automatic detection of personality traits (Regras para formação de grupos de colaboração utilizando deteç̧ão automática de traços de personalidade). Brazilian Journal of Computers in Education (Revista Brasileira de Informática na Educação - RBIE), 28, 273-296. DOI: 10.5753/RBIE.2020.28.0.273 


\section{Introdução}

A ausência de interação física entre estudantes em ambientes virtuais de aprendizagem (AVA) tem sido considerada um grande desafio (Kop, 2011; Everson, 2011; Sadeghi \& Kardan, 2015). Os benefícios desta interação são evidentes tais como estimular a motivação e trocas de conhecimento/experiência e auxiliar na discussão de problemas e dificuldades comuns dos estudantes, contribuindo para um ambiente não só agradável para a aprendizagem, mas principalmente tornando-a efetiva (Alavi, 1994).

Uma das maneiras de promover estas interações é a formação de grupos de colaboração. Segundo (Magnisalis, Demetriadis, \& Karakostas, 2011), a forma como o grupo é formado para a atividade colaborativa é uma maneira de aumentar a probabilidade de ocorrência de interação, já que a composição do grupo, considerando número de integrantes e quem deveria ser agrupado junto, por exemplo) é tida como crucial para desencadear interações produtivas entre os pares. Tais interações são necessárias para a realização de tarefas conjuntas e compartilhamento de conhecimento, características da aprendizagem ativa, na qual o estudante está mais envolvido com o próprio aprendizado. Em ambientes presenciais, esta formação de grupos ocorre de maneira natural sem interferência do professor/tutor, já que os alunos se agrupam conforme conhecimento prévio e afinidades. Entretanto, em ambientes virtuais, estes conhecimentos e afinidades não estão disponíveis, sendo assim, deve ser fornecido algum suporte para identificação de algumas características que permitam aos estudantes e/ou ao ambiente definir grupos.

O modelo Big Five é uma organização hierárquica de traços de personalidade em cinco dimensões: Openness (O), Conscientiousness (C), Extraversion (E), Agreeableness (A) e Neuroticism (N) (De Raad, 2000). O modelo tem sido largamente utilizado na formação de grupos (Sadeghi \& Kardan, 2014; Vindhya \& Mala, 2015; Kelsen \& Liang, 2018; Stadler, Herborn, Mustafic, \& Greiff,' 2019), pois cada dimensão fornece elementos de personalidade compatíveis com as relações sociais e, portanto, com o agrupamento de pessoas. Por exemplo, a extroversão é vista como um fator positivo para a colaboração em um grupo, já que indivíduos extrovertidos gostam de interagir. Por outro lado, indivíduos com alto grau de extroversão, também tendem a se distrair com essa interação. Em um grupo formado apenas por alunos extrovertidos, a meta comum pode acabar sendo negligenciada.

Alto grau de Neuroticism implica em uma pessoa mais preocupada com o que os outros pensam e menos tolerante ao estresse, aumentando a chance desses indivíduos serem mais propensos ao evitamento social. Por outro lado, essa característica de preocupação faz com que cuidem mais das relações sociais, tentando evitar conflitos, o que é positivo para a manutenção de um grupo. Assim, conhecendo tais características, é possível definir uma estratégia de formação de grupos de estudantes de forma a aumentar a chance de interações produtivas para a colaboração.

A utilização de questionários investigativos e questionários de autoavaliação, também chamados de inventários, tem sido frequente no campo de identificação da personalidade (Sadeghi \& Kardan, 2014; Vindhya \& Mala, 2015; Kelsen \& Liang, 2018; Stadler et al., 2019). Outros métodos consistem em estratégias multimodais de identificação para garantir convergência de resultados relacionados ao levantamento dos traços de personalidade, assim como diagnósticos de desordem cognitiva e afetiva, histórico do caso e outros dados como entrevistas, observações de informações ambientais e todo o arcabouço de informações informais que possa ser agregado a testes padrões e questionários (Ekman, 1992; Raento, Oulasvirta, \& Eagle, 2009; Sarkar, Bhatia, Agarwal, \& Li, 2014; Kampman, J. Barezi, Bertero, \& Fung, 2018; Kampman, Siddique, Yang, \& Fung, 2019). 
A utilização dos questionários de avaliação da personalidade na maioria das vezes é extensa e intrusiva (Gosling, Rentfrow, \& Swann Jr., 2003), o que dificulta a extração intencional destas informações. Outras formas mais amigáveis, menos árduas e menos intrusivas, de extração de personalidade, têm sido investigadas com o objetivo de reduzir o impacto causado pela utilização dos questionários, sendo uma delas, a investigação dos traços de personalidade identificados no texto. O processo de identificação da personalidade, e consequente identificação das subjetividades registradas em um texto, de forma computacional, é uma tarefa complexa e exaustiva. Conforme (Munezero, Montero, Sutinen, \& Pajunen, 2014), a subjetividade do ser humano tem forte relação com sentimentos, com as emoções e com as opiniões.

A identificação automática dos traços de personalidade a partir do texto, utilizando métodos computacionais, pode ser observado em (J. W. Pennebaker, Francis, \& Booth, 2001), (Paim, Camati, \& Enembreck, 2016) e (Machado, Longhi, Nunes, \& Pardo, 2015). Desta maneira, é possível evitar o uso dos questionários. Entretanto, observa-se que embora o processamento de linguagem natural (PLN), atualmente, esteja altamente facilitado e fortemente explorado em diferentes aplicações, ainda não permite identificação de características psicológicas com alta acurácia. Então, com o objetivo de constatar as principais causas desta dificuldade, duas equipes de pesquisadores observaram e compararam resultados sobre a identificação destes traços a partir de textos escritos.

Quando boas interações são criadas e mantidas, há maior probabilidade de ocorrer também o compartilhamento de conhecimento (Bozionelos, 2017). Assim, os pesquisadores também conduziram estudos relativos à aplicação dos traços de personalidade detectados nos alunos na formação de grupos, a fim de apoiar a aprendizagem colaborativa. Então, de forma resumida, os objetivos desse trabalho foram apresentar a viabilidade de detectar traços de personalidade automaticamente, através de textos escritos, e demonstrar a influência destes traços na formação de grupos para aprendizagem colaborativa.

Dessa maneira, a pesquisa explorou o uso de PLN para detecção automática de traços de personalidade. Considerando que a maioria dos trabalhos referente a esse aspecto é para a linguagem inglesa, a proposição de ferramentas para linguagem portuguesa (do Brasil) pode ser considerada uma contribuição. Além disso, esse trabalho buscou identificar os algoritmos de aprendizagem mais adequados para lidar com essa detecção, desde que existe um grande número destes algoritmos. A formação de uma base de grupos, que foram qualificados não apenas pelos traços, mas por outros parâmetros, também é uma contribuição, já que a construção de bases com dados reais não é imediata. Entretanto, o diferencial apresentado é a determinação de regras para formação de grupos baseada nos traços. Os estudos encontrados na literatura apenas analisam as características dos grupos, mas não propõem regras para direcionar as formações. Portanto, não é do conhecimento dos autores outro trabalho que apresente resultado semelhante. Dessa forma, a proposta apresentada neste trabalho permite identificar formações que podem apresentar ou não bom desempenho e, então, auxiliar a formação de novos grupos.

Este artigo está organizado como se segue. A Seção 2 apresenta uma breve revisão da literatura relativa ao uso da teoria Big Five na formação de grupos para aprendizagem colaborativa, bem como reflexões sobre o uso de PLN para identificação dos traços. O referencial teórico sobre este estudo é apresentado na Seção 3. A Seção 4 descreve os experimentos de detecção de personalidade conduzidos e compara os resultados obtidos, utilizando tanto bases de dados disponibilizadas para este fim como bases construídas pelos grupos de pesquisa. Também são apresentados os experimentos com grupos em atividade colaborativa e a mineração de regras para formação de grupos. As conclusões e trabalhos futuros se encontram na Seção 5. 


\section{Trabalhos Relacionados}

A composição do grupo é considerada crucial para desencadear interações produtivas entre os membros do grupo (Magnisalis et al., 2011; Sadeghi \& Kardan, 2014; Vindhya \& Mala, 2015; Kelsen \& Liang, 2018; Stadler et al., 2019). Por isso, preocupar-se com a seleção dos indivíduos para compor grupos é uma forma de dar suporte à aprendizagem colaborativa. Uma das formas de conhecer as características dos alunos para aplicar na formação de grupos é avaliar seus traços de personalidade.

Na área da Informática na Educação, uma iniciativa utilizando Personalidade é apresentada pela ferramenta Group Recommender (Nunes, Bezerra, et al., 2010), que possibilita a criação de equipes de trabalho eficientes, levando em consideração as características dos tutores que coordenam as equipes, bem como a similaridade de traços de personalidade dos alunos. Para utilização do sistema, os usuários/alunos têm que responder ao Personality Inventory PV1.0 (Nunes, Moraes, \& Reinert, 2010), do qual se obtém os traços da personalidade e, por meio deste, são gerados os dados necessários para a criação das equipes.

O estudo conduzido por (Carro \& Sanchez-Horreo, 2017) verificou a influência da personalidade e dos estilos de aprendizagem na Educação colaborativa, através de um estudo de caso realizado com estudantes de ensino superior de Engenharia de Computação na Espanha. Os dados dos alunos foram coletados durante a realização de atividades em ambiente virtual, com a aplicação do questionário NEO-FFI, uma versão reduzida do NEO-PI-R (Costa \& Mac Crae, 1985), em conjunto com a identificação de inteligência por meio do teste Primary Mental Abilities (PMA) (Thurstone, 1938) e dos estilos de aprendizagem com o modelo Felder-Silverman (Felder \& Silverman, 1988). Como resultado do trabalho, os autores concluíram que a identificação dos traços de personalidade pode indicar os estudantes ou grupos com potencial de falhar nas atividades e, consequentemente, pode colaborar na construção de sistemas adaptativos com a formação de grupos dinâmicos.

Mesmo a personalidade sendo reconhecida como fator importante na construção de sistemas personalizados, o esforço de capturá-la de forma explícita, utilizando questionários, por exemplo, é indesejado e pode dificultar seu uso em recomendações no mundo real (Wu \& Chen, 2015). Considerando um ambiente virtual de aprendizagem (AVA), onde o aluno não tem a supervisão presencial de um tutor, pode ser difícil conhecer suas características afetivas. Assim, o esforço empregado na construção de ferramentas para extrair as características dos alunos, a partir de informações que podem ser capturadas nos AVAs, permitiria usá-las para apoiar seu processo de aprendizagem.

Tentando enfrentar este desafio, vários trabalhos foram desenvolvidos para extrair conhecimento sobre o aluno, sem a necessidade de aplicação de questionários. No estudo conduzido por (Wen, Yang, \& Rose, 2014), por exemplo, foram realizadas análises de linguística aplicadas às postagens de alunos nos fóruns, para encontrar a relação entre os textos escritos, a motivação e o envolvimento cognitivo do aluno no contexto de cursos on-line massivos (MOOC's). Por sua vez, (Kumar \& Rose, 2014) incorporaram PLN em um agente conversacional, a fim de ajudar os alunos a trabalhar em uma atividade colaborativa e apoiar conversas produtivas.

Além das informações sobre as motivações dos aprendizes, os processos de aprendizagem e a colaboração, pode-se inferir traços de personalidade analisando os textos escritos por um aluno, pois a linguagem é a forma como as pessoas expressam seus pensamentos e emoções. Dessa maneira, a linguagem usualmente carrega parte das características do interlocutor. Então, a partir das palavras que alguém escolhe em uma conversa, é possível encontrar uma relação entre as palavras usadas e a personalidade (Tausczik \& Pennebaker, 2010). 
Durante duas décadas, Dr. James Pennebaker realizou pesquisas sobre a relação entre linguagem, psicologia e saúde. Em conjunto com Martha Francis e Roger Booth, desenvolveu a ferramenta LIWC e o correspondente dicionário. A primeira versão do LIWC surgiu em 1993 como na forma de um estudo exploratório da linguagem e como forma de divulgação do trabalho (Pennebaker et al., 1993). A ferramenta LIWC (Linguistic Inquiry and Word Count) é uma ferramenta de software que permite o cálculo da frequência de ocorrência de diferentes categorias de palavras em um conjunto de textos fornecidos (J. W. Pennebaker et al., 2001). Esta ferramenta tem sua funcionalidade baseada no dicionário homônimo LIWC, que é um léxico contendo cerca de 4.500 palavras, originalmente em inglês, previamente mapeadas em uma ou mais categorias diferentes, dentre as dezenas de categorias existentes neste dicionário.

O trabalho conduzido por Machado et al. (Machado et al., 2015) implementa o Personalitatem Lexicon com o objetivo de identificar os traços de personalidade de um indivíduo a partir da identificação da subjetividade encontrada nos textos produzidos por este indivíduo. $\mathrm{O}$ modelo utilizado por estes autores é baseado no léxico LIWC (J. W. Pennebaker et al., 2001). O experimento foi baseado na avaliação de um conjunto de palavras obtidas em atividades de chat desenvolvidas junto a estudantes de um curso de Pós-graduação em Gestão Pública, associando as palavras com polaridades positivas ou negativas, de acordo com as classes presentes no LIWC, e sua correlação com as dimensões de personalidade definidas pelo modelo Big Five. Os resultados da pesquisa demonstram que existem palavras que indicam os traços de personalidade, mas que ainda não foi possível distingui-las apropriadamente. Na pesquisa desenvolvida por (Paim et al., 2016) é apresentado um método de inferência da personalidade a partir de textos em português, adquiridos na rede social Facebook, usando algoritmos de regressão para construir seus modelos de inferência. Assim, tanto (Machado et al., 2015) quanto (Paim et al., 2016) demonstraram a viabilidade de utilizar métodos para estimar a personalidade por meio de textos escritos em português.

Os algoritmos de aprendizagem de máquina são os mais usados na construção de modelos de detecção automática de traços de personalidade a partir do texto. (Wu \& Chen, 2015) e (Paim et al., 2016), por exemplo, conduziram seus experimentos utilizando algoritmos indutores de regressão. (Tandera, Suhartono, Wongso, \& Prasetio, 2017) e (Majumder, Poria, Gelbukh, \& Cambria, 2017) avaliaram algoritmos de classificação. Já o trabalho desenvolvido por (Tighe, Ureta, Pollo, Cheng, \& de Dios Bulos, 2016) está voltado para a utilização das técnicas de redução de características Information Gain e Principal Component Analysis aplicadas na identificação de personalidade baseado em texto. O trabalho de (Yu \& Markov, 2017) apresenta a identificação da personalidade por meio da utilização de redes neurais fully-connected (FC), convolucionais (CNN) e recorrentes (RNN), utilizando a base de dados denominada myPersonality. Um ponto observado neste trabalho, foi que, além da utilização do texto como fonte de informação para identificação de personalidade, as informações da rede social, disponibilizadas na base myPersonality, foram utilizadas pelos autores, o que representa um conjunto adicional de características no processo de classificação, que em termos educacionais, nem sempre está disponível, e não será tratada no escopo do presente artigo.

A Tabela 1 apresenta um comparativo sobre a acurácia obtida em algumas pesquisas que utilizaram Aprendizado de Máquina para realizar a identificação dos traços de personalidade a partir de textos, utilizando o modelo Big Five. 
Tabela 1: Acurácia Obtida na Identificação dos Traços de Personalidade

\begin{tabular}{|c|c|c|c|c|c|}
\hline ARTIGO & OPN & CON & EXT & AGR & NEU \\
\hline (Tighe et al., 2016) & $61 \%$ & $55 \%$ & $54 \%$ & $57 \%$ & $57 \%$ \\
\hline (Majumder et al., 2017) & $63 \%$ & $57 \%$ & $59 \%$ & $57 \%$ & $59 \%$ \\
\hline (Tandera et al., 2017) & $74 \%$ & $56 \%$ & $65 \%$ & $59 \%$ & $65 \%$ \\
\hline (Yu \& Markov, 2017) & $71 \%$ & $51 \%$ & $61 \%$ & $54 \%$ & $61 \%$ \\
\hline
\end{tabular}

Neste trabalho, além de considerar os algoritmos utilizados em trabalhos relacionados, também foram incluídos nos experimentos outros algoritmos, especificados na subseção 4.1, com o objetivo de selecionar um algoritmo de aprendizagem de máquina adequado para determinar cada um dos traços de personalidade a partir dos textos escritos pelos estudantes.

\section{Formação de Grupos e Personalidade}

Aprendizagem colaborativa envolve a construção conjunta de significado por meio da interação com outros indivíduos e pode ser caracterizada por uma união para atingir uma meta compartilhada. A colaboração efetiva, então, envolve processos sociais por meio dos quais um grupo de alunos trabalham junto para completar uma tarefa (Alavi, 1994), enquanto é exposto a diferentes pontos de vista, questionando sua compreensão inicial e motivando sua aprendizagem. Apoiar os alunos em tarefas colaborativas ajuda, por exemplo, no desenvolvimento de pensamento crítico, autorreflexão e responsabilidade por sua própria aquisição de conhecimento.

Apesar dos benefícios que podem ser atingidos, o processo de colaboração precisa de suporte para ser efetivado. Dentre os vários fatores que podem influenciar o processo de colaboração impedindo, por exemplo, que os alunos interajam entre si, está a composição do grupo (Lai, 2011). Uma vez que a formação do grupo influencia como as pessoas trabalham juntas para alcançar um objetivo, este é um dos aspectos mais importantes a serem considerados, a fim de permitir a aquisição de conhecimento através de atividades colaborativas (Manske, Hecking, Chounta, \& Hoppe, 2015).

Nesta seção apresentamos o referencial teórico que deu suporte ao entendimento da personalidade (Subseção 3.1), a construção de modelos de detecção da personalidade (Subseção 3.2) e o emprego dos traços de personalidade na formação de grupos (Subseção 3.3).

\subsection{Personalidade}

A personalidade pode ser descrita como um conjunto de diferenças individuais que são afetadas pelo desenvolvimento de um indivíduo: valores, atitudes, memórias pessoais, laços sociais, hábitos e habilidades (McAdams \& Olson, 2010; Michel, Shoda, \& Smith, 2004). Uma das principais abordagens utilizadas para o estudo da personalidade humana é a teoria dos traços de personalidade (Kassin, 2003), reconhecida como uma das maiores realizações da Psicologia (Deary, 2009). Estes traços são relativamente estáveis ao longo do tempo, mas diferem de indivíduo para indivíduos e influenciam o seu comportamento.

Os traços de personalidade são características que não podem ser medidos com precisão, mas se um traço de personalidade for relevante, causando diferenças individuais significativas, este será notado (Goldberg, 1981). Com posições próprias, diversos autores indicaram preferências por modelos que contêm um diferente número de dimensões, sendo que um dos modelos mais aceitos e difundidos nesta área é o Big Five Personality Traits (Vinciarelli \& Mohammadi, 2014), que tem sido amplamente utilizado em estudos da personalidade humana e na identificação dos indivíduos em função dos seus traços de personalidade. Tem suas raízes nas 
hipóteses léxicas para identificação de traços de personalidade, sendo baseado nas cinco dimensões:

Openness to Experience: a abertura é o interesse pela arte, emoção, aventura, ideias fora do comum, imaginação, curiosidade, e variedade de experiências;

Conscientiouness: a conscienciosidade, ou meticulosidade, é a tendência para mostrar autodisciplina, orientação para os deveres e para atingir os objetivos;

Extraversion: a extroversão é caracterizada por emoções positivas e pela tendência para procurar estimulação e a companhia dos outros;

Agreeableness: a amabilidade, ou socialização, é a tendência para ser compassivo e cooperante em vez de suspeitoso e antagonista, face aos outros, e;

Neuroticism: o neuroticismo é a tendência para experimentar emoções negativas, como raiva, ansiedade ou depressão.

O Personality Inventory- revised- NEO-PI-R (Costa \& Mac Crae, 1992) pode ser considerado um dos mais relevantes trabalhos na área de identificação manual dos traços de personalidade, pois não somente demonstra grandes propriedades psicométricas, como também consegue acomodar construtores já endereçados pelas métricas existentes de traços de personalidade. Inicialmente utilizando as dimensões Neuroticism, Extraversion e Openness to Experience (NEO), não incluíram as facetas dos recentes fatores Agreeableness e Conscientiouness.

O termo NEO é um acrônimo formado com as iniciais das três dimensões inicialmente incluídas no estudo: Neuroticism, Extraversion e Openness to Experience. Inicialmente, Costa e McCrae incluíram escalas para medição de seis dimensões, conceitualmente derivadas das três dimensões NEO, mas não incluíram as facetas dos fatores Agreeableness e Conscientiouness. Com a publicação do NEO Personality Inventory- revised - NEO-PI-R, o questionário foi ampliado para abranger 240 itens, incluindo estas novas dimensões.

Este questionário é baseado em respostas (cinco pontos da escala Likert) para cada uma das questões, indo de "Discordo Totalmente" até "Concordo Totalmente”. Cada trilha é composta por seis sub-trilhas, cada uma associada a oito questões de avaliação. Um tempo estimado de 45 minutos é previsto para um indivíduo completar este questionário de autoavaliação.

\subsection{Identificação da Personalidade}

A psicologia da linguagem mostra que a escolha das palavras que falamos ou escrevemos não é direcionada somente pelo significado que queremos demonstrar, mas também por fenômenos psicológicos que queremos expressar tais como relações, atitudes, emoções e traços de personalidade (Tausczik \& Pennebaker, 2010), utilizando os questionários NEO-PI-R, por exemplo, considerados como traços verdadeiros da personalidade do indivíduo (Rammstedt \& John, 2007).

Esse reconhecimento utiliza abordagens da Computação Afetiva, Processamento de Sinal Social e outras áreas ligadas a identificação de fenômenos emocionais e sociais através de evidências de comportamento detectáveis automaticamente. Uma dessas abordagens é o reconhecimento automatizado da personalidade a partir de texto. A maioria dos trabalhos realizados nesta área são baseados em Aprendizado de Máquina (Vinciarelli \& Mohammadi, 2014), sendo que o maior desafio tem sido a Representação dos traços de personalidade presentes 
no texto, ou seja, a obtenção das características que permitam a classificação do texto para obtenção dos traços de personalidade do autor de cada texto.

Neste cenário, é verificada a necessidade de técnicas para estruturação do texto, que por natureza é uma fonte de informação não estruturada, de maneira a viabilizar a aplicação de processos computacionais de identificação. Léxicos têm sido empregados para a obtenção de características do texto, através da associação de palavras a certas categorias predefinidas, que podem incluir as emoções, no caso dos léxicos afetivos.

O LIWC (Linguistic Inquiry and Word Count) é um software que permite o cálculo da frequência de ocorrência de diferentes categorias de palavras em um conjunto de textos (J. W. Pennebaker et al., 2001). Esta ferramenta baseia-se no dicionário LIWC, que é um léxico contendo cerca de 4.500 palavras previamente mapeadas em uma ou mais categorias diferentes, dentre as dezenas de categorias existentes neste dicionário. Durante duas décadas, Dr. James Pennebaker realizou pesquisas sobre a relação entre linguagem, psicologia e saúde. Em conjunto com Martha Francis e Roger Booth, desenvolveu a ferramenta LIWC e o correspondente dicionário. Além do dicionário no idioma inglês, a versão do LIWC2007 incorporou uma versão do dicionário em português (Balage Filho, Pardo, \& Aluísio, 2013).

Um outro método utilizado para estruturação de texto é a associação da frequência de ocorrência das palavras encontradas no texto com vetores numéricos. O modelo de predição $n$ gram determina a frequência de ocorrência dos n elementos do texto, gerando as denominações unigram, bigram e trigram, quando estes elementos são associados a uma vizinhança de um, dois ou três elementos, respectivamente. Na área de classificação automática de documentos, a utilização de informações estatísticas da ocorrência de $n$-gram produz relevantes ganhos neste processo (Cavnar \& Trenkle, 1994). Para a obtenção da frequência de ocorrência de cada $n$-gram podem ser utilizadas técnicas como a term frequency-inverse document frequency (TF-IDF), uma medida estatística que tem como objetivo indicar a relevância de um termo em um conjunto de textos.

\subsection{Formação de grupos}

Na literatura, podemos encontrar trabalhos que apontam a influência dos traços de personalidade de cada indivíduo no desempenho de grupos de trabalho (Akhtar, Boustani, Tsivrikos, \& Chamorro-Premuzi, 2015; Vindhya \& Mala, 2015; Stadler et al., 2019). O estudo de (Akhtar et al., 2015), por exemplo, aponta que Conscientiousness influencia diretamente a motivação do indivíduo para atingir uma meta, alta Agreeableness com maior chance de se envolver em trabalho de grupo do que aqueles com baixa Agreeableness, e a Openness, que segundo seus resultados, é o segundo preditor mais forte para o engajamento no trabalho.

Ao avaliar a capacidade dos indivíduos de conectar com novas pessoas ampliando sua rede de convívio social, (Roberts, Wilson, Fedurek, \& Dunbar, 2008) apontam a Extraversion e o Neuroticism como muito significativas para a socialização: a Extraversion como um preditor da intensidade de atividade social, como solicitar ajuda e buscar novas conexões, e o Neuroticism, o oposto. Já (Altanopoulou \& Tselios, 2015) sugerem a distribuição dos indivíduos com alta Conscientiousness entre os grupos, para que eles possam influenciar a habilidade do grupo de cumprir o prazo proposto e, evitar formar grupo com muitos indivíduos com alto grau de Extraversion, uma vez que eles tendem a se distrair mais com a interação social.

(Bozionelos, 2017) aponta ainda a personalidade como tendo um papel importante na formação de laços sociais para a troca de recursos de trabalho. De acordo Neuroticism não tivessem boas interações, tanto indivíduos com alto quanto baixo grau de Extraversion se mostraram bons para a troca de recursos. O mesmo se repete para os níveis baixos de Openness e 
Agreeableness. Aqueles com mais desvantagem em relação à interação para troca de recursos foram aqueles que obtiveram escores intermediários.

Além da forma como cada traço pode afetar um grupo, há também que se considerar a combinação dos traços de cada indivíduo dentro do grupo. O estudo de caso de (Rutherfoord, 2006) aponta grupos heterogêneos, em relação à personalidade, como melhores que os homogêneos. Em grupos homogêneos, é esperado que todos tenham os mesmos pontos fortes e as mesmas fraquezas. Assim, ambos, pontos fortes e fraquezas, são multiplicados pelo número de integrantes. Em um grupo heterogêneo haverá uma maior variedade de habilidade e fraquezas, o que pode ajudar o grupo a gerenciar melhor os problemas que aparecem.

\section{Experimentos e Resultados}

O modelo de identificação dos traços de personalidade dos alunos, utilizando PLN, foi baseado em um processo de Aprendizado de Máquina, no qual as informações textuais, produzidas pelos alunos nas atividades educacionais, foram estruturadas. Esta estruturação consistiu na geração um conjunto numérico representativo de cada texto. Para este processo foi utilizado o léxico LIWC, que permite obter um vetor de características com as informações léxicas e afetivas presentes nestes textos. Como referência para treinamento e teste do processo de classificação, foi também obtida uma identificação dos traços de personalidade de cada aluno, utilizando o questionário BFI44 (John \& Srivastava, 1999), conforme o modelo Big Five. Este questionário de avaliação de personalidade tem 44 questões, em sua versão traduzida para o Português (Andrade, 2008) e cuja validade foi testada no Brasil. Assim, o processo de identificação utilizando somente o texto dos alunos foi comparado com o resultado obtido por meio do questionário, para validar o modelo apresentado.

Assim, nessa seção são apresentados dois conjuntos de experimentos conduzidos para apoiar a formação de grupos. O primeiro conjunto de experimentos, apresentado na Subseção 4.1, visou detectar automaticamente os traços de personalidade dos alunos, para obter indicadores que pudessem facilitar a formação de grupos. A Subseção 4.2 descreve o segundo conjunto de experimentos, tendo como objetivo identificar regras de formação de grupos considerando os traços de personalidade e outros parâmetros, tais como as interações e as notas dos grupos, enquanto realizavam atividade colaborativa. Neste caso, os objetivos foram avaliar a influência dos traços no desempenho do grupo e formar uma base para apoiar a formação de grupos para colaboração.

Para o primeiro conjunto de experimentos, a metodologia consistiu em coletar dos participantes as respostas dadas ao questionário e textos escritos. Assim, em seguida, foram obtidos os traços de personalidade de duas maneiras diferentes: uma através do questionário e outra utilizando os textos. O cálculo dos traços a partir do questionário é feito conforme proposto na teoria do modelo Big Five (John \& Srivastava, 1999). Por outro lado, o cálculo dos traços a partir dos textos são feitos extraindo as palavras significativas, utilizando PLN, em seguida, essas palavras são associadas a categorias conforme dicionário LIWC. Como cada categoria está relacionada a um ou mais traços, a presença de uma tal categoria no texto indica a presença do traço.

Estes experimentos utilizaram o processo de classificação automática, que é um processo computacional que associa ou classifica objetos a determinadas classes, buscando prever uma classe de um novo objeto automaticamente. Para a validação do processo de classificação, são realizados diversos testes a partir de uma base de dados, a qual se tenha conhecimento prévio das classes associadas a estes objetos. Com isto, pode-se obter a acurácia do classificador, ou seja, o 
percentual de objetos identificados corretamente. No caso da identificação dos traços de personalidade, o valor previamente conhecido, que será utilizado na avaliação do classificador, foi obtido com a aplicação de questionários de identificação de personalidade, aos voluntários que participaram dos experimentos.

Como citado na seção 3.2, os algoritmos mais utilizados no reconhecimento automatizado da personalidade a partir de texto são baseados em Aprendizado de Máquina. Contudo, não há uma indicação de qual conjunto de algoritmos é mais adequado para tal classificação. A abordagem adotada neste trabalho foi realizar o processo de treinamento e teste utilizando todos os algoritmos de classificação disponíveis na ferramenta Weka, incluindo também aqueles algoritmos encontrados nos experimentos realizados na literatura. Para seleção dos algoritmos que apresentaram melhor desempenho na classificação, os critérios utilizados foram Acurácia, Erro, Sensibilidade, Especificidade, Valor Previsto S (percentual de valores previstos corretamente como alto escore no traço), Valor Previsto N (percentual de valores previstos corretamente como baixo escore no traço) e Desvio Padrão do Erro.

A base de grupos obtida foi usada como insumo para extração de regras de formação de grupos, que são apresentadas na Subseção 4.3. A metodologia utilizada no segundo conjunto de experimentos considerou os traços previamente obtidos e atividades em grupo. Os traços dos alunos nas bases de dados utilizadas neste experimento foram obtidos somente com a aplicação do questionário BFI-44.

Assim, os participantes realizaram atividades em grupos e o professor avaliou parâmetros como as interações no grupo e atribuiu notas para os resultados apresentados pelos grupos. Em seguida, uma base de grupos foi construída, contendo os traços de cada membro do grupo coletadas com o questionário, e o desempenho do grupo, bom ou ruim, determinado através dos parâmetros avaliados pelo professor. Um algoritmo de mineração de dados, aplicado nesta base, retornou um conjunto de regras, que permitem identificar quando as formações de grupo são promissoras ou não.

\subsection{Detecção de Personalidade}

Os experimentos de detecção de personalidade foram realizados em duas configurações distintas: a primeira envolvendo alunos de ensino superior de Licenciatura em Educação da Universidade Federal Tecnológica Federal do Paraná (UTFPR) e o segundo envolvendo dados coletados na Universidade Federal de Uberlândia (UFU).

No primeiro experimento, os voluntários, alunos regulares em uma disciplina de ensino presencial, com apoio do ambiente Moodle, foram incentivados a utilizar a atividade de Fórum de Discussão. Um conjunto de 45 alunos concordou em participar deste experimento. Foi realizado um experimento em uma instituição brasileira de ensino superior, com três turmas de um curso na área de Educação. Em uma fase inicial, foi realizada uma breve apresentação dos objetivos da pesquisa aos professores responsáveis, sendo definido que seria realizada a aplicação de um questionário de levantamento de traços de personalidade, a ser preenchido pelos alunos. O questionário utilizado foi uma versão do inventário BFI44 (John \& Srivastava, 1999) adaptada e devidamente validada para o idioma português (Andrade, 2008), por meio de uma análise fatorial envolvendo uma amostra de 5.089 voluntários das cinco regiões brasileiras (Silva \& Paraboni, 2018).

Após a coleta dos formulários preenchidos pelos 45 alunos, foi feita a compilação dos resultados e consequente obtenção manual dos traços de personalidade, para cada uma das dimensões do Big Five. Esta obtenção manual dos traços de personalidade é utilizada para validar o método automatizado de identificação, para confrontar os valores Além destes indicadores, 
foram extraídos os conteúdos das mensagens registradas por estes alunos, nas atividades propostas pelos professores, utilizando a atividade de fórum de discussão, no ambiente Moodle. Este procedimento está ilustrado na Figura 1.

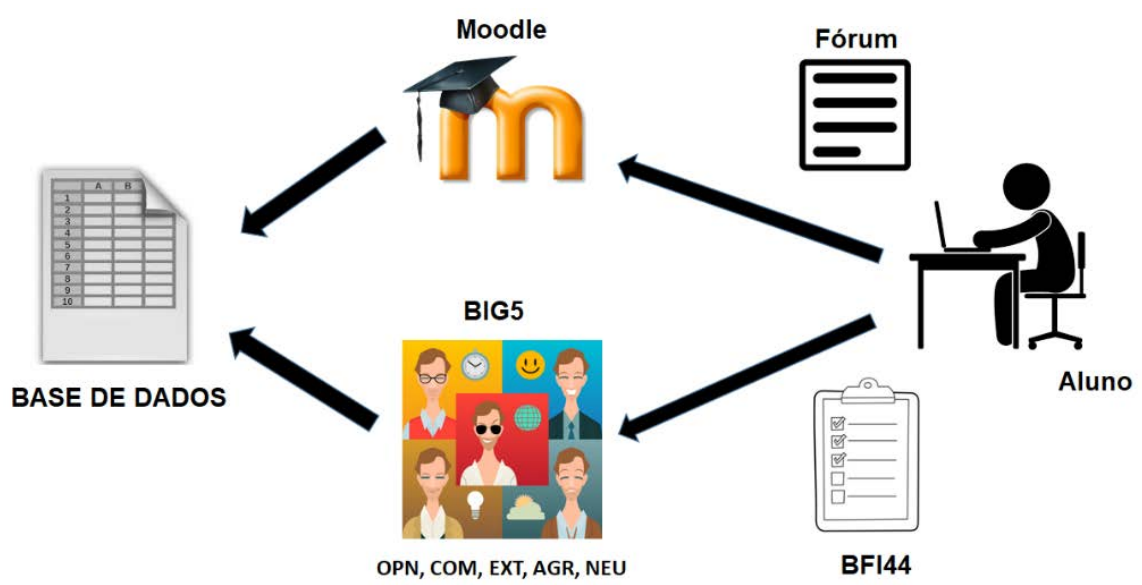

Figura 1: Obtenção da Base de Dados do Experimento 1.

A obtenção dos traços de personalidade de forma manual, utilizando o formulário BFI44,foi realizada somente com o objetivo de construir uma base para a validação inicial do modelo de identificação, contendo o texto dos alunos e a identificação dos traços de personalidade, de acordo com o modelo Big Five, com os valores 0 e 1, correspondendo a presença ou ausência da dimensão específica.

A identificação automatizada do traço de personalidade a partir das palavras presentes nos textos presentes no ambiente Moodle, correspondentes às mensagens inseridas pelos alunos, foi realizada de acordo com o modelo IP3 apresentado por (Buiar, 2018). Para a realização das tarefas de Processamento de Linguagem Natural (PLN) durante este experimento, foi utilizada a biblioteca Natural Language Toolkit — NLTK 3.3 (Bird, Klein, \& Loper, 2009). Com a utilização de um conjunto de programas desenvolvidos durante a condução da presente pesquisa, utilizando a linguagem Python em ambiente Linux, foram realizadas as tarefas de PLN necessárias para a preparação dos textos oriundos das bases, utilizadas no processo de classificação necessário para a identificação dos traços de personalidade.

Em face da quantidade de 45 amostras obtidas junto aos alunos do experimento, foi optado por utilizar a base ESSAYS para treinamento do classificador, obtida por (J. W. Pennebaker \& King, 1999), contendo informações sobre 2.467 voluntários, que incluem textos redigidos por estes, com uma quantidade média de 743 palavras por texto. Esta base já foi validada em diversas pesquisas (Tighe et al., 2016; Majumder et al., 2017; Tandera et al., 2017; Yu \& Markov, 2017) de identificação dos traços de personalidade a partir do texto.

Com a utilização da ferramenta LIWC (versão 2015) (J. Pennebaker, Booth, Boyd, \& Francis, 2015), foi obtida a representação dos textos nas 80 categorias léxicas oferecidas pelos dicionários utilizados pela ferramenta, tanto para a base de treinamento ESSAYS, em inglês, quanto para a base obtida junto aos alunos, em português. Isto foi possível pois a ferramenta LIWC possui dicionários léxicos disponíveis, devidamente validadas, para estes dois idiomas (Balage Filho et al., 2013). 
Os resultados obtidos neste experimento estão apresentados na Tabela 2, onde são indicados os classificadores que obtiveram a melhor Acurácia para cada dimensão de personalidade, bem como os valores de Erro, Sensibilidade (Sen), Especificidade (Esp), Valor Previsto S (VPS), Valor Previsto N (VPN) e Desvio Padrão do Erro (DPErro). O critério kfolds de separação da base em treinamento e teste foi utilizado, com $\mathrm{k}=3$.

Tabela 2: Resultados do Experimento 1.

\begin{tabular}{|c|c|l|l|l|l|l|l|c|}
\hline Dim & Acurácia & Erro & Sens & Esp & VPS & VPN & DPErro & Classificador \\
\hline O & $89 \%$ & $11 \%$ & $97 \%$ & $16 \%$ & $90 \%$ & $16 \%$ & 0,02 & kNN(3) \\
\hline C & $57 \%$ & $43 \%$ & $68 \%$ & $42 \%$ & $62 \%$ & $50 \%$ & 0,03 & AdaBoost \\
\hline E & $66 \%$ & $34 \%$ & $72 \%$ & $60 \%$ & $70 \%$ & $62 \%$ & 0,09 & Random Forest \\
\hline A & $71 \%$ & $29 \%$ & $50 \%$ & $85 \%$ & $72 \%$ & $72 \%$ & 0,08 & Gaussian NB \\
\hline
\end{tabular}

Estes resultados indicam melhores resultados no caso da dimensão Openness e um maior desvio padrão do erro nas três últimas dimensões. Este experimento demonstrou a obtenção de resultados coerentes com os valores encontrados na literatura, para processos de identificação dos traços de personalidade, a partir do texto, mas em função do pequeno número de registros na base original, foi verificada a necessidade de realização de um experimento com uma base maior de alunos.

No segundo experimento, realizado pelos pesquisadores da UFU, os dados utilizados foram coletados de estudantes (nível médio ou superior), que aceitaram participar da pesquisa, por meio de respostas ao questionário e cinco perguntas adicionais que forneceram texto para 0 treinamento do modelo de predição dos traços de personalidade. A coleta das respostas foi feita com o uso de uma escala Likert de 5 pontos. As perguntas adicionais eram cenários nos quais o aluno deveria descrever sua possível resposta ou reação em cada um deles. Os participantes foram orientados a pensar em sua interação com seus amigos ou colegas de turma para responder às perguntas, de forma a permitir capturar linguagem próxima àquela que usa em suas conversas.

A resposta ao questionário foi facultativa e um total de 236 alunos, com idade entre 15 e 56 anos, responderam ao questionário, sendo 106 do sexo feminino e 130 do sexo masculino. Destes, 35 alunos de ensino médio técnico em Informática, 173 de ensino superior e 28 alunos de pós-graduação. O número de alunos com ensino superior corresponde ao total de pessoas que responderam ter ou estar cursando ensino superior. De forma semelhante, pós-graduação considerou tanto os alunos cursando pós-graduação, quanto aqueles que já haviam concluído algum curso de pós-graduação. Os participantes eram alunos da Faculdade de Computação e da Faculdade de Gestão e Negócios da UFU, da Faculdade Pitágoras de Uberlândia, do Centro Universitário do Triângulo e do Instituto Federal Goiano - Campus Campos Belos. Todos os experimentos foram realizados em cursos relacionados à tecnologia da informação, administração e gestão da informação.

Os indicadores obtidos neste segundo experimento são apresentados na Tabela 3, de forma similar ao experimento anterior. Assim como no primeiro experimento, utilizou-se como critério de separação da base em treinamento e teste kfolds, com $\mathrm{k}=3$. Observa-se que quanto maior o valor de VPN (ou VPS), melhor a capacidade do classificador acertar quando prevê N (ou S). Sen e Esp medem a porcentagem da amostra de S e N, respectivamente, que o classificador foi capaz de estimar corretamente. Como a base foi construída por mais amostras de $\mathrm{S}$ do que $\mathrm{N}$ (mais que 80\% de S para Openness, Agreeableness e Conscientiousness), os classificadores que obtiveram maior acurácia foram aqueles onde $\mathrm{Esp}=0$ e VPN=0. Por isso, além de considerar erro e acurácia, os algoritmos da Tabela 3 foram selecionados considerando os valores de Sen, Esp, VPS e VPN. 
Tabela 3: Resultados do Experimento 2.

\begin{tabular}{|c|c|c|c|c|c|c|c|c|}
\hline Dim & Acurácia & Erro & Sen & Esp & VPS & VPN & DPErro & Classificador \\
\hline O & $83 \%$ & $17 \%$ & $95 \%$ & $8 \%$ & $86 \%$ & $25 \%$ & 0.04 & IBK \\
\hline C & $85 \%$ & $15 \%$ & $98 \%$ & $9 \%$ & $87 \%$ & $42 \%$ & 0.03 & RandomForest \\
\hline E & $63 \%$ & $37 \%$ & $74 \%$ & $40 \%$ & $71 \%$ & $45 \%$ & 0.03 & Perceptron \\
\hline A & $85 \%$ & $15 \%$ & $97 \%$ & $12 \%$ & $87 \%$ & $50 \%$ & 0.03 & AdaBoost \\
\hline N & $57 \%$ & $43 \%$ & $51 \%$ & $62 \%$ & $55 \%$ & $59 \%$ & 0.06 & J48 \\
\hline
\end{tabular}

Os melhores algoritmos encontrados para prever os traços de personalidade a partir dos textos fornecidos pelos alunos (texto curtos e informais) neste segundo experimento, considerando todos os critérios de escolha foram: KNN (IBK ${ }^{1}$ ) para Openness, Random Forest para Conscientiousness, Multilayer Perceptron para Extraversion, Ada Boost para Agreeableness, e árvore de decisão (J48) para Neuroticism. Os resultados apresentados na Tabela 3 consideraram os seguintes parâmetros na configuração do Weka: $\mathrm{k}=3$ e distância euclidiana para o KNN; número de árvores numTree $=10$ para o Random Forest; 500 épocas de treinamento, (attribs+classes)/2 camadas escondidas e learning rate de 0,3 para Perceptron; classificador Decision Stump e 10 iterações para o Ada Boost; e para a árvore J48, poda com Minimum Description Length (MDL) para ganho de informação com atributos numéricos.

A Tabela 4 mostra um comparativo entre os resultados obtidos pelos dois experimentos e os resultados obtidos na literatura pesquisada. Ao comparar a acurácia dos experimentos reportados na literatura com os resultados dos experimentos 1 e 2 conduzidos neste trabalho, os resultados dos experimentos 1 e 2 apresentam, em geral, maior acurácia. Além disso, aqueles algoritmos para os quais os demais critérios de avaliação não foram satisfatórios, ainda que obtendo alta acurácia, foram desconsiderados. Os resultados apresentados são mais precisos ao avaliar também a capacidade de cada algoritmo classificar os dados corretamente em cada uma das classes, reduzindo o número de valores previstos errados para cada traço.

Este trabalho, resultado do esforço conjunto de dois grupos de pesquisa para identificação dos traços de personalidade a partir do texto, resultou na seleção de um grupo de algoritmos mais adequados para a identificação dos traços. Como existe um grande número desses algoritmos, e cada trabalho da literatura investigado usa apenas um determinado conjunto deles, por exemplo, somente redes neurais ou somente algoritmos indutores de regressão, uma das contribuições deste trabalho foi a seleção dos algoritmos comparando todos eles para a escolha dos mais adequados.

Em ambos os experimentos 1 e 2, os algoritmos KNN, AdaBoost, Random Forest e Multilayer Perceptron foram identificados como melhores algoritmos para a tarefa de classificação. Além disso, considerando que a maioria dos trabalhos são direcionados para a língua inglesa, a proposição de ferramentas para Português do Brasil pode ser considerada uma contribuição. Assim, a partir dos algoritmos selecionados neste trabalho é possível construir uma ferramenta de detecção dos traços de personalidade a partir de texto escrito em português do Brasil para que esta

\footnotetext{
${ }^{1}$ Esta é a nomenclatura utilizada pelo Weka para KNN
} 
informação possa ser utilizada para fins educacionais, como na formação de grupos para colaboração.

Tabela 4: Comparativo da Acurácia Obtida nos Experimentos.

\begin{tabular}{|c|c|c|c|c|c|}
\hline Referência & O & C & E & A & N \\
\hline (Tighe et al., 2016) & $61 \%$ & $55 \%$ & $54 \%$ & $57 \%$ & $57 \%$ \\
\hline (Majumder et al., 2017) & $63 \%$ & $57 \%$ & $59 \%$ & $57 \%$ & $59 \%$ \\
\hline (Tandera et al., 2017) & $74 \%$ & $56 \%$ & $65 \%$ & $59 \%$ & $65 \%$ \\
\hline (Yu \& Markov, 2017) & $71 \%$ & $51 \%$ & $61 \%$ & $54 \%$ & $61 \%$ \\
\hline Experimento 1 & $89 \%$ & $57 \%$ & $66 \%$ & $71 \%$ & $61 \%$ \\
\hline Experimento 2 & $83 \%$ & $85 \%$ & $63 \%$ & $85 \%$ & $57 \%$ \\
\hline
\end{tabular}

\subsection{Formação de Grupos}

Os experimentos envolvendo a influência de traços de personalidade na formação de grupos foram conduzidos nas turmas presenciais das disciplinas de Engenharia de Software do curso Técnico em Informática para Web do Instituto Federal Goiano - Campus Campos Belos, de Dados e Informações Empresariais e de Análise Financeira da Faculdade de Gestão e Negócios da Universidade Federal de Uberlândia (FAGEN - UFU) e de Gestão da Qualidade de Processo do curso de pós graduação em Engenharia de Software do Centro Universitário do Triângulo. As disciplinas foram ministradas em 2017, totalizando 125 alunos e 38 grupos, contendo de 3 a 5 membros.

Embora esses experimentos tenham sido realizados em sala de aula presencial, isso não afeta os resultados, pois o objetivo foi determinar regras de formação de grupos. Além disso, o cenário desses experimentos exigiu que os alunos apresentassem o resultado da tarefa através de um texto redigido com uso de alguma ferramenta de comunicação, aquela adotada pelo professor, por exemplo, Moodle ou Google Docs. Assim, uma diferença relevante entre presencial e virtual foi contemplada.

Em cada turma, o professor propôs atividades colaborativas, abordando assuntos normalmente previstos para as respectivas aulas, que incluíam a elaboração de texto, após discussão nos grupos. Entretanto, a coleta desses textos foi realizada de maneira diferente, de acordo com o ambiente disponibilizado para redação coletiva do texto. Assim, construiu-se a base de grupos contendo o desempenho de cada grupo observado diretamente pelo professor. A coleta do perfil (traços de personalidade) dos alunos de cada grupo ocorreu por meio da aplicação de um inventário de avaliação de personalidade de 44 questões em sua versão traduzida para o Português (Andrade, 2008). A participação na atividade colaborativa era obrigatória, visto constar da programação de cada disciplina, mas a resposta ao questionário para avaliação dos traços de personalidade era facultativa, não sendo possível determinar os traços de personalidade dos alunos que não quiseram preencher este questionário.

A Tabela 5 apresenta os dados obtidos dos 38 grupos, onde o desempenho de cada grupo foi avaliado de acordo com os seguintes parâmetros: completa tarefa (CT), interação (I) no grupo via conversa, nota atribuída pelo professor (Nota) e a contribuição de todos para a solução (TCS) da tarefa, sendo Tam o número de membros do grupo. A Tabela 6 apresenta o perfil de cada membro dos grupos da Tabela 5. O perfil do aluno é definido pela cadeia de valores na seguinte ordem Openness, Conscientiousness, Extraversion, Agreeableness e Neuroticism. Os valores de cada traço são representados por um caractere que indica uma das faixas para o escore obtido com o questionário. A faixa 1, representada por o, c, e, a e n, indica escore menor que 38,5\%, a faixa 2, representada por -, significa escore entre $38,5 \%$ e $62,5 \%$ inclusive, e a faixa $3(\mathrm{O}, \mathrm{C}, \mathrm{E}, \mathrm{A}$ e $\mathrm{N})$ 
representa escores maiores que 62,5\%. Por exemplo, o aluno com perfil OCE-N obteve escore médio (faixa 2) para Agreeableness e escores altos (faixa 3) para os demais.

Tabela 5: Parâmetros dos grupos.

\begin{tabular}{|c|c|c|c|c|c|}
\hline Grupo & CT & I & Nota & TCS & Tam \\
\hline 1 & 1 & 1 & 0,67 & 1 & 3 \\
\hline 2 & 1 & 1 & 0,73 & 1 & 3 \\
\hline 3 & 0 & 1 & 0,37 & 0 & 3 \\
\hline 4 & 1 & 1 & 0,53 & 1 & 3 \\
\hline 5 & 1 & 1 & 0,83 & 1 & 3 \\
\hline 6 & 1 & 1 & 0,5 & 1 & 2 \\
\hline 7 & 1 & 1 & 0,6 & 1 & 3 \\
\hline 8 & 1 & 1 & 0,5 & 1 & 3 \\
\hline 9 & 1 & 0,3 & 1 & 0 & 5 \\
\hline 10 & 1 & 0,3 & 1 & 0 & 5 \\
\hline 11 & 1 & 0,5 & 1 & 0 & 4 \\
\hline 12 & 1 & 0,5 & 1 & 1 & 3 \\
\hline 13 & 0 & 0,3 & 0 & 0 & 4 \\
\hline 14 & 0 & 0 & 0 & 0 & 1 \\
\hline 15 & 1 & 0,8 & 1 & 1 & 5 \\
\hline 16 & 1 & 0,8 & 1 & 1 & 5 \\
\hline 17 & 1 & 0,8 & 1 & 1 & 3 \\
\hline 18 & 1 & 0,8 & 1 & 1 & 5 \\
\hline 19 & 1 & 1 & 1 & 1 & 5 \\
\hline
\end{tabular}

\begin{tabular}{|c|c|c|c|c|c|}
\hline Grupo & CT & I & Nota & TCS & Tam \\
\hline 20 & 1 & 0,6 & 1 & 1 & 5 \\
\hline 21 & 1 & 0,6 & 0,59 & 0 & 3 \\
\hline 22 & 1 & 0,53 & 0,64 & 0 & 3 \\
\hline 23 & 1 & 0,67 & 0,69 & 0 & 3 \\
\hline 24 & 1 & 0,67 & 0,65 & 1 & 3 \\
\hline 25 & 1 & 0,4 & 0,73 & 1 & 2 \\
\hline 26 & 1 & 1 & 1 & 1 & 3 \\
\hline 27 & 1 & 1 & 1 & 1 & 5 \\
\hline 28 & 1 & 1 & 1 & 1 & 5 \\
\hline 29 & 1 & 1 & 1 & 1 & 3 \\
\hline 30 & 1 & 1 & 1 & 1 & 5 \\
\hline 31 & 1 & 1 & 1 & 1 & 4 \\
\hline 32 & 1 & 0,2 & 1 & 0 & 4 \\
\hline 33 & 1 & 1 & 1 & 1 & 4 \\
\hline 34 & 1 & 1 & 1 & 1 & 4 \\
\hline 35 & 1 & 1 & 1 & 1 & 4 \\
\hline 36 & 1 & 0,8 & 1 & 1 & 3 \\
\hline 37 & 1 & 1 & 1 & 1 & 3 \\
\hline 38 & 1 & 0,8 & 1 & 1 & 4 \\
\hline
\end{tabular}

Na avaliação dos grupos, como o objetivo era formar grupos bons para colaboração, foram considerados como bons grupos aqueles em que todos os membros trabalharam na solução da tarefa proposta e, além disso, havia comunicação que possibilitasse a interação. Os grupos formados por mais de um membro com valores altos para Neuroticism apresentaram bons resultados. Apesar de ser possível observar que dois grupos formados com tal característica obtiveram desempenho ruim (grupos 9 e 11), todos os outros (grupos 1, 2, 6, 8, 16, 18, 27 e 30) apresentaram bons resultados. Baixo Neuroticism também parece influenciar de forma positiva, visto que grupos com baixo grau de Neuroticism, como grupos 5 e 35, ou onde a maioria dos membros com baixo grau de Neuroticism, como os grupos 28 e 33, também formaram bons grupos.

De acordo com a análise do desempenho dos grupos, utilizando as métricas apresentadas na Tabela 5, os grupos formados por membros que tinham valores altos ou baixos de Conscientiousness, Agreeableness e Neuroticism trabalharam bem em conjunto. O mesmo ocorreu quando a maioria do grupo tinha alta Conscientiousness, Openness e Extraversion, bem como naqueles formados por indivíduos que apresentavam baixa ou média Extraversion. Por outro lado, nos grupos onde a maioria tinha escore médio ou médio e alto, alguns foram bons outros não, o que poderia indicar que a performance foi influenciada por outras características dos membros do grupo. 
Tabela 6: Perfil dos alunos dos grupos da Tabela 5.

\begin{tabular}{|c|c|c|c|c|c|}
\hline Grupo & Aluno 1 & Aluno 2 & Aluno 3 & Aluno 4 & Aluno 5 \\
\hline 1 & - C E A N & $-\cdots N$ & $-C-A n$ & & \\
\hline 2 & $\mathrm{OCE}-\mathrm{N}$ & - C E - - & o C E - N & & \\
\hline 3 & $-\mathrm{C}---$ & $-\ldots$ A - & - C E A - & & \\
\hline 4 & OCEAn & O C E A - & O C E A - & & \\
\hline 5 & $-C--n$ & $--E-n$ & OC - - n & & \\
\hline 6 & OCE - N & - c E - N & & & \\
\hline 7 & $\mathrm{O}-\mathrm{E} A-$ & $\ldots$ A n & $\mathrm{OC}--\mathrm{n}$ & & \\
\hline 8 & $--E-N$ & o C - - n & $--\mathrm{E}-\mathrm{N}$ & & \\
\hline 9 & OC - A N & $-\ldots$ A - & $\mathrm{O}-\cdots \mathrm{N}$ & - C - - - & \\
\hline 10 & - CE A n & ? & - . - - & $-\mathrm{C}-\mathrm{-N}$ & $?$ \\
\hline 11 & $\mathrm{OC}-\mathrm{A} \mathrm{N}$ & $-C E-n$ & Oc-A N & $\mathrm{OC}-\mathrm{An}$ & \\
\hline 12 & $\mathrm{OC}-\mathrm{A}-$ & $\mathrm{O}-\mathrm{A}-$ & O c e a - & & \\
\hline 13 & ? & O C E - - & ? & $?$ & \\
\hline 14 & $\ldots$ A - & & & & \\
\hline 15 & O - - A - & ? & O - - - - & $\mathrm{OC}-\mathrm{A}-$ & $--E-N$ \\
\hline 16 & ? & O - E A - & - c E A N & ? & - c E - N \\
\hline 17 & ? & $-\mathrm{C}-\mathrm{-N}$ & - - E A - & & \\
\hline 18 & $\ldots$ & $\mathrm{Oc}-\mathrm{AN}$ & $--\mathrm{E}-\mathrm{N}$ & $\ldots A n$ & - C-A - \\
\hline 19 & OCEAn & - c E A - & o C E A n & - c E A - & $\cdots$ An \\
\hline 20 & - - E A N & - c E A - & Oc - a n & $?$ & $\mathrm{O}-\mathrm{E}-\mathrm{N}$ \\
\hline 21 & OCE A n & $--\mathrm{e}-\mathrm{N}$ & O C - - - & & \\
\hline 22 & $-C-A n$ & OC E - n & $\mathrm{OC}-\mathrm{A} n$ & & \\
\hline 23 & $-\cdots-n$ & O C - - N & - CE A n & & \\
\hline 24 & $-C-A n$ & OC - - n & $-\mathrm{Ce}-\mathrm{N}$ & & \\
\hline 25 & ? & $?$ & & & \\
\hline 26 & O C e - - & - C - - - & $\mathrm{OC}-\mathrm{N}$ & & \\
\hline 27 & $\mathrm{OC}-\mathrm{An}$ & $\mathrm{OCe}-\mathrm{N}$ & $--\mathrm{e}-\mathrm{n}$ & $\cdots-n$ & $\mathrm{O}-\cdots \mathrm{N}$ \\
\hline 28 & $\mathrm{O}-\mathrm{A} \mathrm{n}$ & - C e - - & $-\ldots A n$ & $\ldots A n$ & O- - An \\
\hline 29 & $\mathrm{O}-\mathrm{A} n$ & $\mathrm{O}-\mathrm{-N}$ & O c - - - & & \\
\hline 30 & O - - - & O - . - & - - E A N & $\mathrm{OC}-\mathrm{A} \mathrm{N}$ & O - - - \\
\hline 31 & - CE A - & - - e A - & - - E A - & - - E A - & \\
\hline 32 & $-\mathrm{C}-\mathrm{A}-$ & $-\mathrm{C}-\mathrm{A}-$ & $----n$ & - - - - & \\
\hline 33 & o C E A - & OCEAn & - CE A n & OCEAn & \\
\hline 34 & OC e - - & $-\mathrm{C}---$ & - - e A - & - - E A - & \\
\hline 35 & $\mathrm{O}--\mathrm{A} n$ & $--A n$ & $--A n$ & $\mathrm{O}--\mathrm{A} n$ & \\
\hline 36 & - - - - & $--\mathrm{e}--$ & - C e -- & & \\
\hline 37 & -C-A- & $-\mathrm{C}-\mathrm{A}-$ & 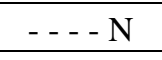 & & \\
\hline 38 & O - . - & - C - - - & - C E A - & - - E A - & \\
\hline
\end{tabular}

Os grupos formados somente por alunos com alto grau de Conscientiousness são bons grupos (por exemplo, grupos 2, 4, 22, 24, 26 e 33). Em geral, ter membros com alta Conscientiousness também parece influenciar positivamente no resultado do grupo. Por outro lado, os grupos 3, 11, 21, 22 e 23, em que nem todos os alunos participaram da solução (TCS=0), também eram formados por maioria de membros com escore alto para Conscientiousness. Nestes grupos, a falta de colaboração pode ser explicada pela combinação dos demais traços. Por exemplo, apesar de serem considerados elementos de ligação em um grupo, aqueles com alto escore em Extraversion e baixo escore em Neuroticism tendem a apresentar excesso de 
entusiasmo, assertividade e autoconfiança, o que pode intimidar aqueles com alto Neuroticism ou baixa Extraversion, como ocorre no grupo 21.

\subsection{Mineração de Regras de Formação de Grupos}

A partir da base de grupos construída, tornou-se viável minerar regras de agrupamento, através do algoritmo Predictive Apriori (Scheffer, 2001), utilizando a classe Predictive Apriori do Weka (Frank, Hall, \& Witten, 2016). A fim de manter certa homogeneidade da base para aplicação do algoritmo, grupos em que os traços da maioria dos membros eram desconhecidos ou que continham apenas um membro, foram desconsiderados. Desde que os grupos restantes eram compostos de 2 a 5 indivíduos, foi necessária uma representação capaz de fornecer uma entrada com o mesmo número de atributos para o algoritmo.

Essa representação foi obtida como resultado do pré-processamento dos dados, que atribuiu a cada um dos cinco traços de personalidade dos membros do grupo um valor conforme as regras descritas na Tabela 7. A primeira coluna (Escores no Grupo) refere-se aos escores apresentados pelos membros do grupo e a segunda coluna (Traço) o valor que é atribuído ao traço.

Tabela 7: Valores do resumo dos traços de personalidade.

\begin{tabular}{|c|c|}
\hline Traço & Escores no Grupo \\
\hline A & Todos ou maioria dos membros obtiveram escore alto no traço \\
\hline B & Todos ou maioria dos membros obtiveram escore baixo no traço \\
\hline M & Todos ou maioria dos membros obtiveram escore médio no traço \\
\hline MB & Parte dos alunos têm escore médio e a outra parte, escore baixo no traço \\
\hline MA & Parte dos alunos têm escore médio e a outra parte, escore alto no traço \\
\hline AB & Todos os membros têm escores alto ou baixo no traço \\
\hline AMB & Os membros têm escores alto, médio e baixo no traço \\
\hline
\end{tabular}

Além do mapeamento dos traços de personalidade, a entrada foi composta também pelas métricas de grupo tamanho, prazo e o desempenho do grupo (GP). O valor GP atribuído pode ser BOM ou RUIM de acordo com o desempenho do grupo (DP) dado por DP $=0.4 * \mathrm{TCS}+$ $0.1 *$ Nota $+0.3 * \mathrm{I}+0.2 * \mathrm{CT}$, conforme definido em (Ferreira, 2018). Esse valor corresponde à média ponderada das métricas de grupo. Se DP > 0.5, o grupo é classificado como GP=BOM, e GP=RUIM, caso contrário.

Os pesos mais altos foram aplicados a TCS e I porque são as métricas que dão indícios sobre o quanto os membros do grupo estão interagindo entre si. Por exemplo, se um membro do grupo não trabalha junto com os demais para resolver a tarefa, TCS assume valor zero e, por consequência, faz com que o desempenho calculado do grupo seja menor. CT e Nota recebem pesos menores, mas não são completamente descartadas do cálculo, pois também ajudam a medir a qualidade do grupo. Ainda que o grupo tenha muita interação, espera-se que um bom grupo alcance nota satisfatória e consiga concluir a tarefa proposta.

A partir desses dados, o algoritmo foi executado sobre a base atualizada com os grupos provenientes do experimento de formação de grupos. A execução do Predictive Apriori resultou em 57 regras. No entanto, algumas das regras foram extraídas da base com baixa precisão. Todas as regras com precisão menor que $60 \%$ foram removidas da lista de regras apresentadas na Tabela 8. 
Toda regra retornada possui uma condição que, quando satisfeita, indica se o grupo tem mais chance de obter desempenho Bom ou Ruim. Na regra 2, por exemplo, a condição diz que se o grupo for formado por indivíduos com escore $M$ para Agreeableness $(A=M)$, então o desempenho do grupo na atividade colaborativa deverá ser bom (GP=BOM). Essa regra foi extraída da base com mais de $98 \%$ de precisão (Precisão). A regra 16 da Tabela 8 foi extraída com precisão de mais de $95 \%$ e diz que, se o grupo tem quatro membros (tamanho=4) e o prazo é de uma semana (prazo=W), esse grupo pode falhar $(\mathrm{GP}=\mathrm{RUIM})$. Assim, cada uma delas pode ser usada para verificar se um grupo já formado funciona bem ou se pode ter desempenho ruim.

Tabela 8: Regras extraídas da base de 38 grupos que fizeram tarefas colaborativa.

\begin{tabular}{|c|c|c|}
\hline Id & Regra & Precisão \\
\hline 1 & $\mathrm{E}=\mathrm{MB}==>\mathrm{BOM}$ & $98,60 \%$ \\
\hline 2 & $\mathrm{~A}=\mathrm{M}==>\mathrm{BOM}$ & $98,60 \%$ \\
\hline 3 & $\mathrm{~N}=\mathrm{MB}==>\mathrm{BOM}$ & $97,97 \%$ \\
\hline 4 & tamanho=3 O=MA ==> BOM & $97,97 \%$ \\
\hline 5 & $\mathrm{E}=\mathrm{A}==>\mathrm{BOM}$ & $97,39 \%$ \\
\hline 6 & $\mathrm{~N}=\mathrm{MA}==>\mathrm{BOM}$ & $97,39 \%$ \\
\hline 7 & tamanho=3 C=A ==> BOM & $97,39 \%$ \\
\hline 8 & tamanho=3 N=AB ==> BOM & $97,39 \%$ \\
\hline 9 & $\mathrm{O}=\mathrm{A}==>\mathrm{BOM}$ & $96,44 \%$ \\
\hline 10 & $\mathrm{O}=\mathrm{AMB}==>\mathrm{BOM}$ & $96,44 \%$ \\
\hline 11 & $\mathrm{C}=\mathrm{AMB}==>\mathrm{BOM}$ & $96,44 \%$ \\
\hline 12 & $\mathrm{E}=\mathrm{M}==>\mathrm{BOM}$ & $96,44 \%$ \\
\hline 13 & $\mathrm{~A}=\mathrm{A}==>\mathrm{BOM}$ & $96,44 \%$ \\
\hline 14 & tamanho=3 ==> BOM & $95,04 \%$ \\
\hline 15 & $\mathrm{~A}=\mathrm{AMB}==>\mathrm{BOM}$ & $94,75 \%$ \\
\hline 16 & tamanho=4 $\mathrm{prazo}=\mathrm{W}==>\mathrm{RUIM}$ & $94,75 \%$ \\
\hline 17 & tamanho=4 $\mathrm{E}=\mathrm{AMB}==>\mathrm{BOM}$ & $94,75 \%$ \\
\hline 18 & tamanho=4 E=MA ==> RUIM & $94,75 \%$ \\
\hline 19 & tamanho=4 N=M ==> BOM & $94,75 \%$ \\
\hline
\end{tabular}

\begin{tabular}{|l|c|c|}
\hline Id & Regra & Precisão \\
\hline 20 & tamanho=5 prazo=W ==> RUIM & $94,75 \%$ \\
\hline 21 & prazo=C ==> BOM & $84,32 \%$ \\
\hline 22 & $\mathrm{C}=\mathrm{A}==>\mathrm{BOM}$ & $79,75 \%$ \\
\hline 23 & $\mathrm{~N}=\mathrm{M}==>\mathrm{BOM}$ & $79,75 \%$ \\
\hline 24 & $\mathrm{C}=\mathrm{MA}==>\mathrm{BOM}$ & $76,93 \%$ \\
\hline 25 & prazo=W ==> RUIM & $75,83 \%$ \\
\hline 26 & $\mathrm{O}=\mathrm{M}==>\mathrm{BOM}$ & $75,83 \%$ \\
\hline 27 & $\mathrm{O}=\mathrm{MA}==>\mathrm{BOM}$ & $73,39 \%$ \\
\hline 28 & tamanho=5 $\mathrm{E}=\mathrm{MA}==>\mathrm{BOM}$ & $70,85 \%$ \\
\hline 29 & $\mathrm{~A}=\mathrm{MA} \mathrm{N}=\mathrm{AMB}==>\mathrm{BOM}$ & $70,85 \%$ \\
\hline 30 & $\mathrm{~A}=\mathrm{MA}==>\mathrm{BOM}$ & $68,79 \%$ \\
\hline 31 & $\mathrm{~N}=\mathrm{AB}==>\mathrm{BOM}$ & $67,23 \%$ \\
\hline 32 & tamanho=5 ==> BOM & $65,01 \%$ \\
\hline 33 & $\mathrm{E}=\mathrm{MA}==>\mathrm{BOM}$ & $65,01 \%$ \\
\hline 34 & tamanho=4 ==> BOM & $64,43 \%$ \\
\hline 35 & $\mathrm{E}=\mathrm{AMB}==>\mathrm{BOM}$ & $64,14 \%$ \\
\hline 36 & tamanho=4 N=AB ==> RUIM & $64,14 \%$ \\
\hline 37 & $\mathrm{O}=\mathrm{MA} \mathrm{E}=\mathrm{MA} \mathrm{N}=\mathrm{AB}==>\mathrm{RUIM}$ & $64,14 \%$ \\
\hline 38 & $\mathrm{~N}=\mathrm{AMB}==>\mathrm{BOM}$ & $63,54 \%$ \\
\hline
\end{tabular}

As regras extraídas podem ser empregadas tanto na avaliação de grupos já formados ou na formação de novos grupos. Na avaliação dos grupos, as regras podem ser usadas para verificar se há chance de o grupo ter desempenho bom ou ruim, e se há necessidade de desfazê-lo. Por exemplo, tomando o grupo 1 da Tabela 5 como grupo a ser avaliado, de acordo com o perfil de seus membros, poderíamos associá-lo às regras 8 (tamanho 3 e indivíduos apresentando Neuroticism alto ou baixo), 24 (indivíduos apresentando Conscientiousness alta ou média) e 26 (todos com Openness média), todas as três apontando o desempenho presumido do grupo como bom.

O grupo 11 da Tabela 5 pode ser associado à regra 18, que indica que grupos de 4 membros com membros apresentando Extraversion alta ou média, são grupos com chance de falhar, como foi de fato observado no resultado do grupo 11. Por outro lado, o fato de um grupo poder ser associado a uma regra que aponta desempenho ruim, não necessariamente significa que o desempenho será ruim, mas é um alerta que o professor pode usar para reavaliar a formação de um grupo.

Quanto à formação de novos grupos, uma vez que se conhece os perfis dos alunos de uma turma, seja por meio de um questionário ou utilizando outro método de detecção dos traços de personalidade, é possível empregar as regras na formação de novos grupos. Os grupos podem ser 
formados associando cada aluno às regras para as quais seu perfil é compatível, iniciando pelas regras de maior precisão e que tem como consequente GP=BOM. O perfil do aluno é considerado compatível com uma regra quando suas características permitem a participação em um grupo como aquele descrito pela regra. Por exemplo, considerando a regra 1, são indivíduos com perfis compatíveis àqueles que têm Extraversion média ou baixa. Portanto, um aluno altamente extrovertido não poderia ser associado à regra 1.

Depois de associar as regras ao perfil de cada aluno, grupos podem ser formados com alunos que foram associados às mesmas regras. Aqui é preciso tomar cuidado ao formar os grupos para que ainda continuem mantendo as características descritas nas regras. Há a possibilidade, por exemplo, de todos os indivíduos associados à regra 1 serem somente aqueles com Extraversion média. Assim, não haverá possibilidade de formar um grupo com E=MB. Neste caso, deve-se descartar a formação do grupo com a regra 1.

As regras de agrupamento apresentadas foram extraídas de base de dados dos grupos construída a partir de grupos e alunos reais. A formação de uma base de grupos qualificados pelos traços, e outros parâmetros de grupo, é uma das contribuições desse trabalho, já que a construção de bases com dados reais não é imediata. Além da base de dados, as regras também são uma contribuição deste trabalho. As regras, extraídas com alta precisão da base, fornecem direcionamento para formar novos grupos para colaboração e, também, para identificar formações que podem ou não apresentar um bom desempenho, apoiando o professor na tarefa de reorganizar os grupos.

\section{Conclusão}

Este artigo descreveu a aplicação do modelo Big Five na formação de grupos de alunos, utilizando textos em linguagem natural na identificação dos traços de personalidade, que apresenta um grande desafio computacional. As técnicas mais utilizadas estão baseadas no aprendizado de máquina e em uma série de estratégias de representação do texto de forma estruturada, a fim de permitir a realização de processos de classificação. Mesmo com grandes esforços na utilização de classificadores mais complexos e técnicas de representação mais aprimoradas, a melhoria nos indicadores de classificação, em especial a acurácia, não avançam na mesma proporção.

A dificuldade da obtenção de bases de treinamento para os classificadores, os ruídos presentes no processo de identificação a partir de questionários de avaliação e a quantidade de palavras presentes nos textos utilizados interferem de forma direta nos resultados. Nos experimentos realizados, o uso de $n$-gram durante o processamento da linguagem natural foi uma alternativa explorada, visando melhorar a identificação das categorias do dicionário. Entretanto, os resultados não justificaram a complexidade deste acréscimo.

Em um experimento inicial foi verificada a aplicação de um processo de identificação em ambiente educacional, mesmo com uma quantidade reduzida de alunos que participaram do experimento. A partir desta referência foi conduzido um segundo experimento em maior escala. Os resultados demonstraram que uma metodologia de identificação da personalidade dos alunos pode oferecer suporte relevante para tutores e estudantes em AVAs. Contudo, considerando-se que a quantidade e a diversidade de texto são de suma importância para a classificação dos traços, os resultados encontrados foram promissores.

Uma vez que é possível detectar os traços de personalidade por meio dos textos escritos pelos alunos e avaliar seu desempenho, é possível usar tais informações em uma estratégia que usa os traços de personalidade para formação de grupos. Visando definir tal estratégia, conduzimos um experimento com grupos de no máximo 5 alunos trabalhando em atividades 
colaborativas e avaliamos seu desempenho, de acordo com os traços de personalidade dos alunos de cada grupo, detectado por meio da aplicação de um questionário. O desempenho e os traços de personalidade dos alunos dos grupos foram empregados na construção de uma base de dados de grupos, a qual foi utilizada para extração de regras de formação de grupos, por meio de mineração de dados. Como resultado, obtivemos um conjunto de regras de formação de grupos que podem ser empregadas na construção de bons grupos para colaboração.

Conforme indicado na avaliação dos resultados, as regras extraídas podem ser usadas tanto para avaliar a qualidade de um grupo formado, para que o professor possa ter indícios de quando precisa desfazer um grupo, quanto para formação de novos grupos. Assim, os resultados deste trabalho também abrem espaço para o desenvolvimento de uma ferramenta para formação de grupos que integra a detecção dos traços de personalidade do aluno, a partir de textos, e os utiliza para formar grupos, aplicando as regras para formação de grupos apresentadas neste trabalho.

Ainda como perspectiva futura, os pesquisadores destes grupos acreditam que melhorias significativas da qualidade dos grupos formados estão associadas ao uso de outras métricas que, em conjunto com o modelo Big Five, permitam a identificação de outros elementos relevantes para formação de grupos, como aqueles considerados na avaliação dos grupos conduzida neste trabalho. Assim, é possível melhorar a qualidade e precisão das regras de formação de grupos e empregá-las de maneira mais efetiva na formação de bons grupos para colaboração.

\section{Agradecimentos}

Os pesquisadores agradecem o suporte (Código de Financiamento 001) da Coordenação de Aperfeiçoamento de Pessoal de Nível Superior - CAPES - para a realização das pesquisas que resultaram neste trabalho.

\section{Referências}

Akhtar, R., Boustani, L., Tsivrikos, D., \& Chamorro-Premuzi, T. (2015). The engageable personality: Personality and trait EI as predictors of work engagement. Personality and Individual Differences, 73, 44-49. doi: 10.1016/j.paid.2014.08.040 [GS Search]

Alavi, M. (1994). Computer-Mediated Collaborative Learning: An Empirical Evaluation. Journal MIS Quarterly, 18, 159-174. doi:10.2307/249763 [GS Search]

Altanopoulou, P., \& Tselios, N. (2015). How does personality affect wiki-mediated learning? Proceedings of International Conference on Interactive Mobile and Communication Technologies and Learning, (pp. 16-18). doi:10.1109/IMCTL.2015.7359546 [GS Search]

Andrade, J. M. (7 de 2008). Evidências de Validade do Inventário dos Cinco Grandes Fatores de Personalidade para o Brasil. Ph.D. dissertation, Instituto de Psicologia - Universidade de Brasília. Fonte: https://core.ac.uk/download/pdf/33530474.pdf

Balage Filho, P. P., Pardo, T. A., \& Aluísio, S. M. (2013). An Evaluation of the Brazilian portuguese LIWC Dictionary for Sentiment Analysis. 9th Brazilian Synposium in Information and Human Language Technology, 215-219.

Bird, S., Klein, E., \& Loper, E. (2009). Natural language processing with Python: analyzing text with the natural language toolkit. " O'Reilly Media, Inc.". [GS Search] 
Bozionelos, G. (2017). The relationship of the big-five with workplace network resources: More quadratic than linear. Personality and Individual Differences, 104, 374-378. doi: 10.1016/j.paid.2016.08.036 [GS Search]

Buiar, J. A. (9 de 2018). Modelo computacional e sua implementação para identificação de perfil de personalidade baseado em textos educacionais. Ph.D. dissertation, Universidade Federal do Paraná. Fonte: https://hdl.handle.net/1884/57936

Carro, R. M., \& Sanchez-Horreo, V. (2017). The effect of personality and learning styles on individual and collaborative learning: Obtaining criteria for adaptation. Global Engineering Education Conference (EDUCON), 2017 IEEE (pp. 1585-1590). IEEE. doi:10.1109/EDUCON.2017.7943060 [GS Search]

Cavnar, W. B., \& Trenkle, J. M. (1994). N-gram-based text categorization. Ann arbor mi, 48113, 161-175. [GS Search]

Costa, P. T., \& Mac Crae, R. R. (1985). The NEO personality inventory. Psychological Assessment Resources Odessa, FL. [GS Search]

Costa, P. T., \& Mac Crae, R. R. (1992). Neo personality inventory-revised (NEO PI-R). Psychological Assessment Resources Odessa, FL. [GS Search]

De Raad, B. (2000). The Big Five Personality Factors: The psycholexical approach to personality. Hogrefe \& Huber Publishers. [GS Search]

Deary, I. J. (2009). The trait approach to personality. The Cambridge handbook of personality psychology, 1, 89-109. [GS Search]

Ekman, P. (1992). An argument for basic emotions. Cognition \& emotion, 6, 169-200. doi: $\underline{10.1080 / 02699939208411068}$ [GS Search]

Everson, M. (2011). What can you gain by teaching online? eLearn Magazine, 2011, 3. doi: $\underline{10.1145 / 1925037.1925058}$ [GS Search]

Felder, R. M., \& Silverman, L. K. (1 de 1988). Learning and teaching styles in engineering education. Engineering education, 78, 674-681. [GS Search]

Ferreira, T. B. (8 de 2018). Raciocínio Baseado em Casos para apoiar a formação de grupos baseada nos traços de personalidade do modelo Big Five. Universidade Federal de Uberlândia. Universidade Federal de Uberlândia. Fonte: http://clyde.dr.ufu.br/handle/123456789/23284

Frank, E., Hall, M. A., \& Witten, I. H. (2016). The WEKA Workbench Online Appendix for Data Mining: Practical Machine Learning Tools and Techniques (4 ed.). Morgan Kaufmann. [GS Search]

Goldberg, L. R. (1981). Language and individual differences: The search for universals in personality lexicons. Review of personality and social psychology, 2, 141-165. [GS Search]

Gosling, S. D., Rentfrow, P. J., \& Swann Jr., W. B. (12 de 2003). A very brief measure of the BigFive personality domains. Journal of Research in Personality, 37, 504-528. doi:10.1016/S0092-6566(03)00046-1 [GS Search]

John, O., \& Srivastava, S. (1999). The Big Five trait taxonomy: History, measurement, and theoretical perspectives. Handbook of personality: Theory and research, 2, 102-138. [GS Search] 
Kampman, O., J. Barezi, E., Bertero, D., \& Fung, P. (7 de 2018). Investigating Audio, Video, and Text Fusion Methods for End-to-End Automatic Personality Prediction. Proceedings of the 56th Annual Meeting of the Association for Computational Linguistics (Volume 2: Short Papers) (pp. 606-611). Melbourne: Association for Computational Linguistics. [GS Search]

Kampman, O., Siddique, F. B., Yang, Y., \& Fung, P. (2019). Adapting a virtual agent to user personality. Em Advanced Social Interaction with Agents (pp. 111-118). Springer. doi: $\underline{10.1007 / 978-3-319-92108-213}$ [GS Search]

Kassin, S. M. (2003). Essentials of psychology. Prentice Hall. [GS Search]

Kelsen, B. A., \& Liang, H.-Y. (2018). Role of the Big Five Personality Traits and Motivation in Predicting Performance in Collaborative Presentations. Psychological reports. doi: $\underline{10.1177 / 0033294118795139}$ [GS Search]

Kop, R. (3 de 2011). The challenges to connectivist learning on open online networks: Learning experiences during a massive open online course. The International Review of Research in Open and Distance Learning, 12.doi:10.19173/irrodl.v12i3.882 [GS Search]

Kumar, R., \& Rose, C. P. (2014). Triggering Effective Social Support for Online Groups. ACM Transactions on Interactive Systems, 3, artigo 24 doi:10.1145/2499672 [GS Search]

Lai, E. R. (2 de 2011). Collaboration: A Literature Review. Pearson. [GS Search]

Machado, A. A., Longhi, M. T., Nunes, M. A., \& Pardo, T. A. (2015). Personalitatem Lexicon: Um Léxico em Português Brasileiro para Mineração de traços de Personalidade em Textos. Anais do XXVI Simpósio Brasileiro de Informática na Educação, (pp. 1122-1126). doi:10.5753/cbie.sbie.2015.1122 [GS Search]

Magnisalis, I., Demetriadis, S., \& Karakostas, A. (2011). Adaptive and Intelligent Systems for Collaboration Learning Support: A Review of the Field. IEEE Transactions on Learning Technologies, 4, 5-20. doi:10.1109/TLT.2011.2 [GS Search]

Majumder, N., Poria, S., Gelbukh, A., \& Cambria, E. (2017). Deep learning-based document modeling for personality detection from text. IEEE Intelligent Systems, 32, 74-79. doi:10.1109/MIS.2017.23 [GS Search]

Manske, S., Hecking, T., Chounta, I. A., \& Hoppe, H. U. (2015). Using differences to make a difference: a study on heterogeneity of learning groups. Proceedings of International Conference on Computer Supported Collaborative Learning, (pp. 182-189). [GS Search]

McAdams, D. P., \& Olson, B. D. (2010). Personality development: Continuity and change over the life course. Annual review of psychology, 61, 517-542. doi:10.1146/annurev.psych.093008.100507 [GS Search]

Michel, W., Shoda, Y., \& Smith, R. E. (2004). Introduction to personality: Toward an integration. New York: John Wiley. [GS Search]

Munezero, M. D., Montero, C. S., Sutinen, E., \& Pajunen, J. (2014). Are they different? Affect, feeling, emotion, sentiment, and opinion detection in text. IEEE transactions on affective computing, 5, 101-111. doi:10.1109/TAFFC.2014.2317187 [GS Search]

Nunes, M. A., Bezerra, J. S., Reinert, D., Moraes, D., Silva, E. P., \& Pereira, A. J. (2010). Computação Afetiva e sua influência na personalização de Ambientes Educacionais: gerando equipes compatíveis para uso em AVAs na EaD. Educação E Ciberespaço: Estudos, Propostas E Desafios. Aracaju: Virtus Editora, 1, 308-347. [GS Search] 
Nunes, M. A., Moraes, D. B., \& Reinert, D. (2010). Personality Inventory-PV 1.0 (Portuguese Version). Instituto Nacional de Propriedade Industrial.(software register INPI-12093-0). [GS Search]

Paim, A., Camati, R., \& Enembreck, F. (2016). Inferência de Personalidade a partir de textos em Português utilizando Léxico Linguístico e Aprendizagem de Máquina. Anais do XIII Encontro Nacional de Inteligência Artificial e Computacional, (pp. 481-492). [GS Search]

Pennebaker, J. W., \& King, L. A. (1999, 12). Linguistic styles: Language use as an individual difference. Journal of Personality and Social Psychology, 77, 1296-1312. doi:10.1037/0022-3514.77.6.1296 [GS Search]

Pennebaker, J. W., Booth, R. J., Boyd, R. L., \& Francis, M. E. (2015). Linguistic inquiry and word count: LIWC2015. Austin, TX, USA: Pennebaker Conglomerates. [GS Search]

Pennebaker, J. W., Francis, M. E., \& Booth, R. J. (2001). Linguistic inquiry and word count: LIWC 2001. Mahway: Lawrence Erlbaum Associates, 71, 2001. [GS Search]

Raento, M., Oulasvirta, A., \& Eagle, N. (2009). Smartphones: An emerging tool for social scientists. Sociological methods \& research, 37, 426-454. doi: $\underline{10.1177 / 0049124108330005}$ [GS Search]

Rammstedt, B., \& John, O. P. (2 de 2007). Measuring personality in one minute or less: A 10item short version of the Big Five Inventory in English and German. Journal of Research in Personality, 41, 203-212 doi:10.1016/j.jrp.2006.02.001 [GS Search]

Roberts, S. G., Wilson, R., Fedurek, P., \& Dunbar, R. I. (2008). Individual differences and personal social network size and structure. Personality and Individual Differences, 44, 954-964 doi:10.1016/j.paid.2007.10.033 [GS Search]

Rutherfoord, R. H. (2006). Using Personality Inventories to Form Teams for Class Projects - A Case Study. Proceedings of SIGITE '06 Proceedings of the 7th conference on Information Technology Education, (pp. 9-14). doi:10.1145/1168812.1168817 [GS Search]

Sadeghi, H., \& Kardan, A. A. (2014). Toward effective group formation in computer-supported collaborative learning. Interactive Learning Environments, 24, 1-14. doi: $\underline{10.1080 / 10494820.2013 .851090}$ [GS Search]

Sadegui, H., \& Kardan, A. (2015). A novel justice-based linear model for optimal learner group formation in computer-supported collaborative learning environments. Computers in Human Behavior, 48, 436-447. doi:10.1016/j.chb.2015.020 [GS Search]

Sarkar, C., Bhatia, S., Agarwal, A., \& Li, J. (2014). Feature analysis for computational personality recognition using youtube personality data set. Proceedings of the 2014 ACM multi media on workshop on computational personality recognition, (pp. 11-14). doi:10.1145/2659522.2659528 [GS Search]

Scheffer, T. (2001). Finding Association Rules That Trade Support Optimally against Confidence. 5th European Conference on Principles of Data Mining and Knowledge Discovery (pp. 424-435). Berlin: Springer Berlin Heidelberg. doi:10.1007/3-540-44794-635 [GS Search]

Silva, B., \& Paraboni, I. (2018). Learning personality traits from Facebook text. IEEE Latin America Transactions, 16, 1256-1262. doi:10.1109/TLA.2018.8362165 [GS Search]

Stadler, M., Herborn, K., Mustafić, M., \& Greiff, S. (2019). Computer-Based Collaborative Problem Solving in PISA 2015 and the Role of Personality. Journal of Intelligence, 7. doi: $\underline{10.3390 / j i n t e l l i g e n c e 7030015}$ [GS Search] 
Tandera, T., Suhartono, D., Wongso, R., \& Prasetio, Y. L. (10 de 2017). Personality Prediction System from Facebook Users. Procedia Computer Science, 116, 604-611. doi:10.1016/j.procs.2017.10.016 [GS Search]

Tausczik, Y. R., \& Pennebaker, J. W. (2010). The Psychological Meaning of Words: LIWC and Computerized Text Analysis Methods. Journal of Language and Social Psychology, 29, 24-54.doi:10.1177/0261927X09351676 [GS Search]

Thurstone, L. L. (1938). Primary mental abilities. Psychometric monographs. [GS Search]

Tighe, E. P., Ureta, J. C., Pollo, B. A., Cheng, C. K., \& Dios Bulos, R. (2016). Personality Trait Classification of Essays with the Application of Feature Reduction. SAAIP@ IJCAI, (pp. 22-28). [GS Search]

Vinciarelli, A., \& Mohammadi, G. (2014). A survey of personality computing. IEEE Transactions on Affective Computing, 5, 273-291. doi:10.1109/TAFFC.2014.2330816 [GS Search]

Wen, M., Yang, D., \& Rose, C. (2014). Linguistic Reflections of Student Engagement in Massive Open Online Courses. Proceedings of the International Conference on Weblogs and Social Media. [GS Search]

Wu, W., \& Chen, L. (2015). Implicit Acquisition of User Personality for Augmenting Movie Recommendations. Proceedings of International Conference on User Modeling, Adaptation and Personalization, (pp. 302-314). doi: $\underline{10.1007 / 978-3-319-20267-925}$ [GS Search]

Yu, J., \& Markov, K. (2017). Deep Learning based Personality Recognition from Facebook Status Updates. The 8th International Conference on Awareness Science and Technology. doi: $\underline{10.1109 / I C A w S T .2017 .8256484 ~[G S ~ S e a r c h] ~}$ 